\title{
AUTO-GERENCIAMENTO DE FALHAS NO CONTEXTO DE AMBIENTES INTELIGENTES E ASSISTIDOS: UMA ABORDAGEM BASEADA EM REDES BAYESIANAS
}

\author{
Lilia S. Da S. Morais, Maria A. Silva, Camila H. S. Oliveira \\ Centro de Ciências e Tecnologia - Universidade Federal do Cariri (UFCA) \\ Av. Ten. Raimundo Rocha, 1639 - Cidade Universitária \\ Juazeiro do Norte - CE - Brazil \\ \{lilia.suyane, adriana.silva\}@aluno.ufca.edu.br, camila.oliveira@ufca.edu.br
}

\begin{abstract}
The elderly population is a demographic group in constant expansion, which leads to the emergence of problems associated with this population health care. Thus, looking for ensuring a better quality of life, many countries have invested in solutions that allow the elderly to have more autonomy in their daily live activities. Ambient Assisted Living (AAL) is one solution that presents great results in helping individuals who have some limitations, and in unburdening the responsibilities of their caregivers. In this paper, we propose a framework for fault detection and diagnosis that seeks to guarantee the quality of services offered in those environments. Our framework uses the Bayesian Networks approach to model AALs and in the process of detecting failures in the physical networks employed in AALs.
\end{abstract}

Resumo - Com o envelhecimento da população cresce o interesse em soluções que permitam que os idosos com limitações cognitivas tenham mais autonomia no seu cotidiano e uma melhor qualidade de vida. Ambiente Inteligentes e Assistidos (AIA) têm sido usados em vários países com o obejtivo de oferecer assistência a esta população que está em pleno crescimento em todo o mundo. Neste artigo, propomos um framework de detecção e diagnóstico de falhas que busca garantir a qualidade dos serviços oferecidos nestes ambientes. Nosso framework usa a abordagem de Redes Bayesianas na construção de modelos que serão utilizados na detecção de falhas dos dispositivos empregados nos AIAs.

\section{Introdução}

O envelhecimento da população é um fenômeno que vem avançando e trazendo consigo problemas sérios decorrentes da perda de autonomia apresentada pela população idosa. Embora, no Brasil, ele ocorra de forma menos acelerada quando comparado com a situação em países desenvolvidos [Brito 2008] [Yunes 1971], o processo de transição demográfica, resultante da diminuição das taxas de natalidade e mortalidade de nossa população, prova que a antes jovem população brasileira começou o seu processo de envelhecimento [Braga et al. 2008].

Entre os inúmeros problemas que essa mudança na pirâmide etária brasileira pode causar destacamos o aumento no número de casos de problemas cognitivos que aparecem 
no processo natural de envelhecimento, mas também decorrentes de doenças como o Alzheimer. Neste contexto, Ambientes Inteligentes e Assistidos (AIAs) vêm sendo propostos com o intuito de melhorar a qualidade de vida de pessoas idosas, assim como de seus cuidadores [Radziszewski et al. 2016][ Sun et al. 2009].

A ideia é que em um ambiente inteligente, onde serviços de assistência sejam implementados, os idosos possam viver de forma mais segura e manter sua autonomia e independência. Segundo [Al-Shaqi et al. 2016], soluções de AIAs centradas no usuário permitem que eles vivam mais tempo em suas residências o que contribue para um maior bem-estar dos mesmos.

A base dos serviços implementados em AIAs é os dispositivos que constituem as Redes Sensores e Atuadores Sem Fio (RSASF). Essa rede é composta de dispositivos que contêm nós sensores implantados em uma determinada área para detectar e monitorar os fenômenos físicos que ocorrem no local. Uma das características dessas redes é que são propícias a falhas como: quebra, interferência de comunicação por conta de outros elementos no ambiente e falta de energia. Essas falhas comprometem a transmissão e a coleta de informações [Gutiérrez et al. 2019], o que resulta na perda da eficiência de seu funcionamento provocando assim um possível problema ao indivíduo que depende do AIA no seu cotidiano. Portanto, dada a natureza sensível dos serviços oferecidos nos AIAs, a detecção e diagnóstico de falhas nessas redes são muito importantes.

O problema de diagnóstico de falhas em RSASFs tem sido amplamente estudado, visto que, com o desenvolvimento da IoT, esses dispositivos se tornaram mais acessíveis e usados para implementar uma ampla gama de serviços. Na literatura, nós encontramos diferentes abordagens usadas para resolver esse problema. Em [Jadav et al. 2017], os autores usam um modelo de lógica fuzzy para detectar nós com falha e diagnosticar três tipos de problemas: problema no circuito do transmissor, no circuito de recepção e na bateria. Em [He et al. 2018], um sistema de crenças é proposto para realizar diagnósticos em rede de sensores sem fio. Em [Zidi et al. 2018], os autores propõem um SVM (método de classificação) para gerar uma função de decisão usada pelo líder do cluster para identificar um sensor com defeito. Zhang [Zhang et al. 2018] fornece uma cobertura adicional de estudos sobre o diagnóstico de falhas em RSASFs. Embora esses trabalhos tenham propostos soluções para resolver o problema de dispositivos defeituosos em RSASFs, eles não levam em consideração as características específicas dos AIAs.

Neste artigo, propomos um framework que tem por objetivo implementar um mecanismo de detecção e diagnóstico em RSASFs que leva em consideração as especificidades dos AIAs onde essas redes estão sendo empregadas. A construção do framework consiste em duas etapas. Na primeira, uma Rede Bayesiana (RB) personalizada com base nos dispositivos que compõem o AIA é construída a partir do contexto onde o AIA será implementado e da assistência oferecida. Na segunda, a estrutura de RB criada na primeira etapa é utilizada para a realização de inferências que são usadas como base para detectar e diagnosticar falhas. Nesse artigo, apresentamos especificamente o desenvolvimento do algoritmo utilizado para a modelagem dos AIAs.

\section{Metodologia}

A metodologia adotada no desenvolvimento deste artigo se baseia na abordagem de Redes Bayesianas. Uma RB é definida como um grafo acíclico no qual os nós representam as variáveis aleatórias usadas para modelar o AIA e as arestas representam as relações de 
interdependência entre os nós. Um exemplo muito usado ao falar sobre Redes Bayesianas é o do Alarme. Considere um alarme, muito confiável, que detecta intrusões dentro de uma casa e que também dispara em caso de terremoto. Considere também dois vizinhos, João e Maria, que irão telefonar caso o alarme dispare. João sempre liga quando ouve o alarme, mas algumas vezes ele confunde o telefone com o alarme e também liga nesses casos. Maria, por outro lado, gosta de ouvir música alta e, às vezes, não escuta o alarme.

Portanto, é possível notar a presença das seguintes variáveis (nós) no modelo: Assalto (B), Terremoto (E), Disparo do Alarme (A), Ligação de João (J) e Ligação de Maria (M). Além disso, as setas entre B/A e E/A representam que, neste caso, B e E têm influência direta em A. Esse tipo de influência é matematicamente representada pelas Tabelas de Probabilidades Condicionais (TPCs) definidas para cada nó no modelo Bayesiano. Assim, a partir do modelo representado na Figura 1, é possível calcular a distribuição de probabilidade de qualquer variável. Por exemplo, a probabilidade do alarme ter tocado sem ocorrência de ladrão ou terremoto, dado que João ligou, pode ser calculada através da probabilidade condicional $\mathrm{P}(\mathrm{A} \mid \mathrm{J})$.

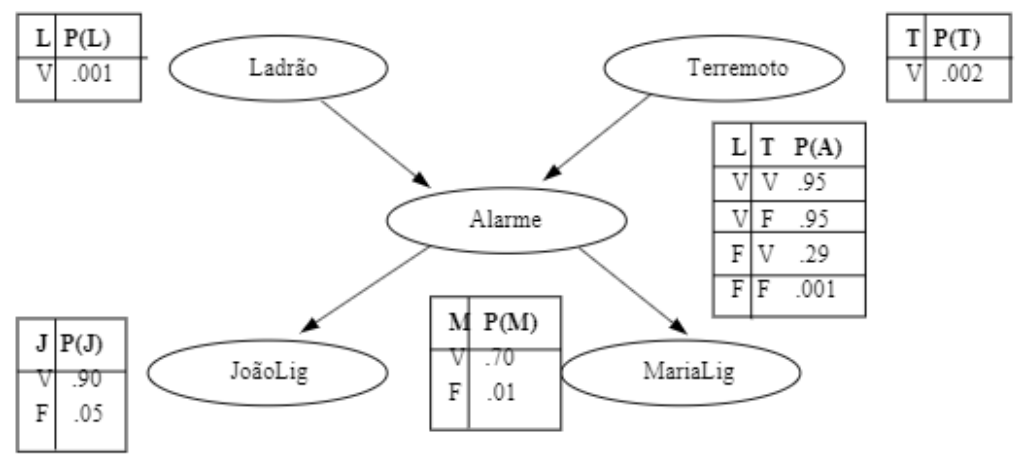

Figure 1. Rede Bayesiana representando o exemplo do alarme.

A abordagem Bayesiana apresenta uma característica muito importante para nosso projeto. Como vimos no exemplo anterior, a inferência realizada no modelo permite o cálculo da distribuição de probabilidade de uma variável mesmo quando não se tem conhecimento completo da situação em um determinado momento. Como trabalhamos com as RSASFs, e estas são propícias à falha, essa característica das RBs faz dela a abordagem ideal para ser utilizada no nosso framework.

Nosso framework propõe que a detecção e o diagnóstico de falhas em AIAs sejam baseados em um modelo Bayesiano personalizado construído a partir de informações fornecidas pelos usuários, como a assistência requerida e o contexto onde a aplicação será empregada. Uma vez que a RB é construída, um motor de inferência e os dados fornecido pelo ambiente (através dos sensores) são usados para identificar e diagnosticar as falhas na rede. No entanto, para que a distribuição probabilística retornada como resultado pelo motor de inferência seja precisa e possa levar a diagnósticos confiáveis, os modelos Bayesianos usados pelo framework devem conseguir representar fielmente os AIAs que eles estão modelando. Por isso, o Bayesian Network Construction Algorithm (BNCA) está no centro do funcionamento do nosso framework é o componente que nos interessa neste artigo.

O BNCA tem como principal objetivo construir a partir de informações do AIA uma RB que modela o contexto do ambiente, levando em consideração tanto os dispositivos físicos utilizados (sensores e atuadores) como informações da casa e da 
assistência que será implementada. Como mostrado na Figura 2, BNCA precisa de dois arquivos de entrada, o primeiro sendo o arquivo Regras que é constituído por todas as relações possíveis entre os dispositivos que podem compor a rede de sensores e atuadores. E o segundo, o arquivo Cenário que é elaborado de acordo com a assistência solicitada e a descrição do ambiente físico onde o AIA será implementado.

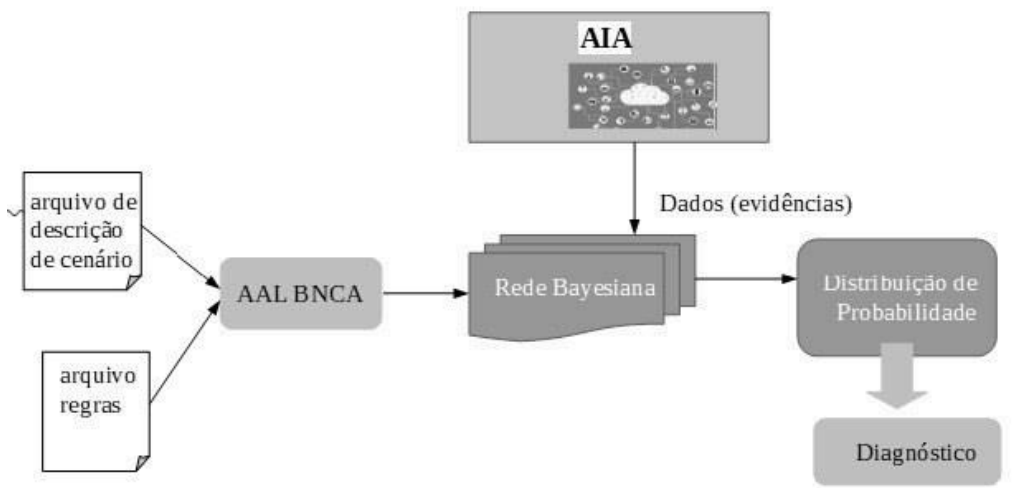

Figure 2. Funcionamento do BNCA dentro do framework.

No arquivo Regras, as relações são determinadas de acordo com as funcionalidades associadas aos dispositivos em um AIA. Dessa forma, ao criar relações entre os nós representando os dispositivos físicos e os nós representando as funcionalidades do sistema, BNCA estabelece as relações de interdependência entres os elementos definidos no modelo.

$\mathrm{O}$ arquivo Cenário é utilizado para fornecer ao framework informações sobre o contexto onde o AIA será inserido. Ele contém uma tabela onde detalhes como a presença de porta/janela, sensores que serão instalados, valores utilizados para representar seus estados, fonte de energia, identificação, e a ação que eles terão no ambiente são definidos. Assim, a partir dessas informações, BNCA pode gerar uma lista com todos os nós necessários para modelar um AIA específico.

BNCA trabalha em três estágios:

1. a criação de nós a partir do cenário analisado;

2. a identificação das relações pai-filho possíveis;

3. a criação das Tabelas de Probabilidades Condicionais.

$\mathrm{Na}$ primeira etapa, BNCA cria um arquivo nós onde são gravados os nós observáveis (sensores que serão distribuídos em um determinado ambiente), e os nós não observáveis (variáveis ligadas com as funcionalidades implementadas pela assistência). A criação desses nós é baseada no arquivo Cenário que é definido como uma entrada do BNCA. Para facilitar o entendimento, considere o exemplo A onde uma assistência, cuja a única finalidade é acender a luz do quarto quando uma presença é detectada, é implementada. A Tabela 1 representa um arquivo Cenário possível para este AIA. Neste caso, BNCA criaria um arquivo nós com os seguintes nós: movementdetected, daynight, movement, lamp, night. 
Tabela 1. Arquivo cenário do exemplo A.

\begin{tabular}{|c|c|c|c|c|c|c|c|}
\hline room & $\begin{array}{c}\text { nb__ } \\
\text { room }\end{array}$ & room_feature & sensor_type & sensor_value & sensor_id & action & source_en \\
\hline bedroom & hall & door_hall & movement & 0,1 & movement & $\begin{array}{c}\text { movement- } \\
\text { detected_bedro } \\
\text { om }\end{array}$ & battery \\
\hline bedroom & hall & door_hall & lamp & 0,1 & lampl & $\begin{array}{c}\text { turnon_lamp- } \\
\text { bedroom_perso } \\
\text { n_ }\end{array}$ & battery \\
\hline bedroom & hall & door_hall & light & 0,1 & light1 & $\begin{array}{c}\text { illuminate_bedr } \\
\text { oom_person }\end{array}$ & battery \\
\hline
\end{tabular}

No segundo estágio, BNCA utiliza o arquivo nós, criado na primeira etapa do código, e o arquivo Regras. $\mathrm{O}$ arquivo Regras têm suas linhas definidas na forma filho = lista de possíveis pais. BNCA percorre cada linha do arquivo Regras e quando o filho da linha em questão corresponder a um nó do arquivo nós, uma relação de pai-filho é criada. Vale ressaltar que os filhos podem ter mais de um pai, ou seja, mais de uma variável é capaz de mudar o valor de estado associado a um nó.

O terceiro estágio consiste na criação das TPCs. As TPCs são criadas a partir de experimentos realizados em um AIA real. Os dados coletados do AIA uma vez que a rede de dispositivos IoT é instalada são usados para calcular a tabela correspondente de cada variável do modelo criado para o AIA em questão.

\section{Resultados}

Para analisarmos se BNCA é capaz de gerar RB confiáveis e fiéis ao AIA que ele modela, propomos um caso de estudo onde o algoritmo será aplicado, e analisaremos o resultado gerado pelo mesmo.

Cenário de aplicação: Uma mulher de 64 anos diagnosticada com Alzheimer e que apresenta episódios de sonambulismo. A filha deseja que sua mãe seja guiada para a saída do quarto de forma segura durante seus episódios. Assumimos nesse estudo de caso que o quarto possui acesso a um corredor e que não possui nenhuma janela.

Dada a assistência requerida pelo usuário, um AIA poderia ser composto da seguinte forma: um sensor de movimento a ser instalado perto da cama para conferir se há movimento no quarto; um sensor beam a ser instalado na porta do quarto para verificar quando ela sair do quarto; um sensor de pressão a ser colocado embaixo do colchão para detectar quando ela levanta da cama; um sensor de luminosidade para detectar a presença de luz no ambiente; e dois atuadores, o lamp e o lightpath, que ajudarão a guiá-la até a saída do quarto iluminando o caminho.

Suponha que a partir da descrição dos dispositivos e da assistência pedida uma plataforma de AIA cria o arquivo Cenário que será usado como entrada para BNCA. Em nosso estudo de caso assumimos o arquivo Cenário apresentado na Tabela 2: 
Tabela 2. Arquivo Cenário do estudo de caso.

\begin{tabular}{|c|c|c|c|c|c|c|c|}
\hline room & $\begin{array}{c}\text { nb_- } \\
\text { room }\end{array}$ & room_feature & sensor_type & $\begin{array}{c}\text { sensor_ } \\
\text { value }\end{array}$ & sensor_id & action & source_en \\
\hline bedrrom & hall & door_hall & movement & 0,1 & MotionSensor & $\begin{array}{c}\text { movement- } \\
\text { detected_bedroom }\end{array}$ & battery \\
\hline bedroom & hall & door_hall & beam & 0,1 & BeamSensor & $\begin{array}{c}\text { door-crossed_bedroom- } \\
\text { hall_detected }\end{array}$ & cable \\
\hline bedroom & hall & door_hall & pressure & 0,1 & PressureSensor & detected_person_bed & cable \\
\hline bedroom & hall & door_hall & light & 0,1 & LightSensor & illuminate_bedroom_per & cable \\
\hline bedroom & hall & door_hall & lightpath & 0,1 & Lightpath & turnon_lightpath_person & cable \\
\hline bedroom & hall & door_hall & lamp & 0,1 & Lamp & turnon_lamp_person & cable \\
\hline
\end{tabular}

Uma vez que BNCA recebe o arquivo Cenário, ele cria um arquivo nós com todas as variáveis que a RB modelando nosso estudo de caso terá. Neste caso, o arquivo teria os seguintes nós: movement, beam, pressure, light, lightpath, lamp, movementdetected, doorcrossed e daynight.

Em seguida, BNCA utiliza o arquivo Rules para a partir dos nós criar as ligações que conectam as variáveis na $\mathrm{RB}$. Como resultado, BNCA gera o modelo Bayesiano mostrado na Figura 3.

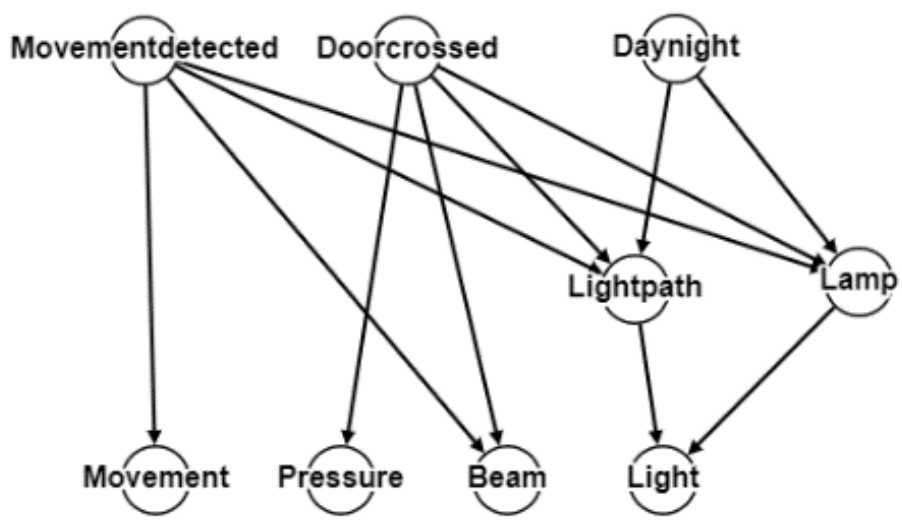

Figura 3. RB do estudo de caso.

Análise do cenário: A senhora de 64 anos apresenta um episódio de sonambulismo, levanta-se da sua cama e sai do seu quarto. Logo, temos que o AIA deve executar a assistência de guiá-la até a porta. Supondo então que o sensor pressão detecta que ela saiu da cama e que o sensor de movimento confirma que há movimento no quarto, os atuadores lamp e lightpath recebem sinal de ativação e são ligados para guiá-la até a porta. Uma vez que ela sai do quarto, o sensor beam é ativado e as luzes permanecem ligadas até que ela retorne a sua cama. Durante os episódios de sonambulismo os dados capturados pelos sensores alimentam uma base de dados que serão utilizadas pelo framework para detectar e diagnosticar possíveis falhas na rede.

A eficiência do framework para detectar e diagnosticar as falhas depende do quão preciso é o modelo Bayesiano gerado pelo BNCA. Dada a RB da Figura 3, pode-se observar que as relações entre os nós modelam exatamente a assistência que está sendo 
oferecida no AIA em questão. Veja, por exemplo, os nós movementdetected e doorcrossed que impactam diretamente no lamp e no lightpath. Essas relações modelam as ações que desencadeiam a ativação da assistência no AIA. No entanto, para que o modelo seja validado é preciso avaliá-lo também no cenário em que ocorre uma falha. Para isso, assuma a seguinte situação:

Cenário de falha: A mulher levanta no meio da noite, sai do quarto, mas um dos atuadores, o lamp, não funciona. Considere que ela levante no período da noite, o que implica que a assistência será ativada. Dessa forma, quando a mulher levanta da cama temos que o sensor de movimento capta o seu movimento e o sensor de pressão detecta que a mesma levantou da cama. Então, a assistência é ativada acionando o lightpath e o lamp que são os atuadores utilizados para iluminar o caminho de saída do quarto. A senhora sai do quarto e o sensor beam detecta que a mulher atravessou a porta. No entanto, como supomos o lamp com defeito, apenas o lightpath será ligado. Os dados gerados pelos dispositivos durante a execução deste cenário são armazenados na base de dados do framework.

Se a RB modelando o AIA instalado no quarto é fiel a rede e ao ambiente de instalação do AIA, o framework deve ser capaz de detectar o problema no dispositivo lamp. Para isso, ele usa o motor de inferência e os dados coletados durante a assistência para detectar se tem algum problema na rede de dispositivos. Neste estudo de caso, podemos observar na RB (Figura 3) que tanto lamp quanto lighpath estão diretamente conectados com os nós movimentdetected, doorcrossed e daynight. Assim, o framework pode fazer uma query para saber qual a probabilidade de lamp está ligado (ativo) dado que lightpath está ligado, e movementdetected, doorcrossed e daynigth foram ativados.

O framework possui essas informações porque quando os dispositivos mudam de estado eles enviam atualizações para a base de dados. Dada a quantidade de evidência passada na query, o motor de inferência retorna com alta probabilidade que o estado do dispositivo lamp deveria ser 1 (ativo). Mas na base de dados a única informação sobre lamp é que ele não foi ativado pois não chegou nenhuma atualização. Assim, o framework deduz que o dispositivo lamp deve estar com problema e deverá fazer análises mais aprofundadas para achar o diagnóstico preciso, ou seja, o tipo de problema apresentado pelo dispositivo. Essa etapa não está no escopo deste artigo e será fruto da continuação da nossa pesquisa.

A RB gerada pelo BNCA para o cenário de estudo de caso mostra que ele é capaz de gerar modelos representativos do ambiente físico que compõe um AIA. Mostramos que as relações necessárias para ativação do cenário assim como aquelas fundamentais para a detecção de falhas estão presentes no modelo, o que confirma a eficiência do algoritmo em modelar um sistema AIA em particular levando em consideração todas suas especificidades.

\section{Conclusão}

Neste artigo, desenvolvemos e implementamos um dos componentes do nosso framework de detecção e diagnóstico de falhas baseado em RB. A implementação do BNCA é parte fundamental do framework dado que se trata do componente responsável por modelar o sistema AIA no qual um serviço de assistência será empregado. A principal contribuição deste artigo foi a construção do algoritmo que permite a criação de grafos nos quais as relações de dependência encontradas entre os sensores do cenário são representadas. 
A estrutura criada poderá assim ser utilizada para analisar as mudanças de estado dos dispositivos através de queries executadas por meio de um motor de inferência Bayesiano. Mostramos através de um estudo de caso que dado um cenário de aplicação, BNCA cria uma RB capaz de representar todos os aspectos importantes de um AIA, como os dispositivos, o ambiente físico e a assistência prestada ao usuário. O próximo passo consiste em implementar um componente do framework chamado Monitor que será responsável por detectar e diagnosticar as falhas da rede através de inferências executadas no modelo gerado pelo BNCA.

\section{Referências}

Brito, F. (2008) “Transição demográfica e desigualdades sociais no Brasil”. Rev. bras. estud. popul., São Paulo, v.25, n.1, p.5-26, jun.

Yunes, J. (1971 "A dinâmica populacional dos países desenvolvidos e subdesenvolvidos”. Rev. Saúde Pública, São Paulo, v.5, n.1, p.129-150, jun.

Braga, S., Filho, A., Silveira, R., Guimarães. (2008) “As Políticas Públicas para os Idosos no Brasil: A Cidadania no Envelhecimento", Encontro de Administração Pública e Governança (ENAPG). Salvador, Bahia.

Radziszewski, R., Ngankam, H.K., Pigot, H., Grégoire, V., Lorrain, D., \& Giroux, S. (2016) "An ambient assisted living nighttime wandering system for elderly", Proceedings of the 18th International Conference on Information Integration and Webbased Applications and Services.

Sun H., Florio V. D., Gui N. and Blondia C. (2009) "Promises and Challenges of Ambient Assisted Living Systems", Sixth International Conference on Information Technology: New Generations, 2009, pp. 1201-1207, doi: 10.1109/ITNG.2009.169.

Al-Shaqi, R., Mourshed, M. and Rezgui, Y. (2016) "Progress in ambient assisted systems for independent living by the elderly", Springerplus. 2016 May 14;5:624. doi: 10.1186/s40064-016-2272-8. PMID: 27330890; PMCID: PMC4870543.

Gutiérrez, S. and Ponce, H. (2019) “An Intelligent Failure Detection on a Wireless Sensor Network for Indoor Climate Conditions. Sensors”, Sensors 19.4 (2019): 854.

Jadav P. and Babu V. K. (2017) "Fuzzy logic based faulty node detection in wireless sensor network,", International Conference on Communication and Signal Processing (ICCSP), pp. 0390-0394.

He W., Qiao P. L., Zhou Z. J., Hu G. Y., Feng Z. C. and Wei H. (2018) “A new beliefrule-based method for fault diagnosis of wireless sensor network", IEEE Access, vol. PP, no. 99, pp. 1-1, 2018.

Zidi S., Moulahi T. and Alaya B. (2018) "Fault detection in wireless sensor networks through svm classifier”, IEEE Sensors Journal, vol. 18, no. 1,pp. 340-347, Jan 2018.

Zhang Z., Mehmood A., Shu L., Huo Z., Zhang Y. and Mukherjee M. (2018) "A survey on fault diagnosis in wireless sensor networks", IEEE Access, vol. PP, no. 99, pp. 1-1, 2018. 